\title{
Abscopal effect of high-dose-rate brachytherapy on pelvic bone metastases from renal cell carcinoma: a case report
}

\author{
Gen Suzuki, MD, PhD!, Koji Masui, MD!, Hideya Yamazaki, MD, PhD!, Tadashi Takenaka, RTT', Syunsuke Asai, MD!, \\ Hidefumi Taniguchi, MD, PhD², Terukazu Nakamura, MD, PhD², Osamu Ukimura, MD, PhD², Kei Yamada, MD, PhD' \\ 'Department of Radiology, Kyoto Prefectural University Graduate School of Medical Science, Kamigyo-ku, Kyoto, Japan, ${ }^{2}$ Department of \\ Urology, Kyoto Prefectural University Prefectural University Graduate School of Medical Science, Kamigyo-ku, Kyoto, Japan
}

\begin{abstract}
Radiation therapy is considered an optimal partner for immunotherapies. Several pre-clinical studies have demonstrated that regression of distant metastases, at remote non-irradiated sites of the body, termed the "abscopal effect", can be achieved by an appropriate timing and combination of radiation with immunotherapy. However, nearly all pre-clinical and clinical studies evaluating a combination of radiation and immunotherapies have used external beam radiation therapy. We present in this case report, the abscopal effect observed in a 30-year-old Japanese woman with metastatic renal cell carcinoma after the treatment with high-dose-rate interstitial brachytherapy combined with nivolumab. This is the first published report demonstrating an abscopal effect following brachytherapy for human malignancy. Our case indicates that immuno-oncology effects are not limited to external beam irradiation regimens as they can also be attained by brachytherapy.

J Contemp Brachytherapy 2019; II, 5: 458-46l DOI: https://doi.org/10.5114/jcb.2019.89365
\end{abstract}

Key words: brachytherapy, immunotherapy, metastatic renal cell carcinoma, nivolumab, radiation, abscopal effect.

\section{Purpose}

The abscopal effect refers to the regression of non-irradiated metastatic lesions at distant areas from the primary site of irradiation. In the last decade, the introduction of immune checkpoint inhibitors (ICIs), such as the PD-1 inhibitor, has revolutionized therapeutic strategy for a wide variety of advanced malignancies. Radiotherapy holds a significant promise as a potential partner for combination therapies. Particularly, combining radiation and immunotherapy in the clinical setting has shown to improve outcomes in several types of malignant tumors $[1,2,3,4]$.

The abscopal effect has only rarely been observed prior to the widespread application of immunotherapy, and an increase in prevalence was observed with the recent introduction of ICIs [4]. Thus far, nearly all preclinical and clinical studies evaluating radiation therapy in combination with immunotherapies have used external beam radiotherapy (EBRT), and little is known about the efficacy of immune modulation combined with brachytherapy [5]. Herein, we describe a patient with intractable metastases of renal cell carcinoma (RCC) who exhibited the abscopal effect after a combination treatment with nivolumab and high-dose-rate interstitial brachytherapy
(HDR-ISBT). To the best of our knowledge, this is the first published report demonstrating the abscopal effect following brachytherapy for human malignancy.

\section{Case presentation}

A 30-year-old woman underwent radical nephrectomy for clinically localized left RCC in 1998, and was pathologically diagnosed with clear cell RCC, pT2b and Fuhrman grade 2. Bilateral lung metastases were found eight years after nephrectomy, and both lesions were surgically removed, followed by postoperative interferon treatment. However, by 2010 she underwent five lung resections and also one radiofrequency ablation, and the patient achieved long-term response with adjuvant sunitinib. In May 2013, a right ovarian metastasis was detected, which was surgically excised. In July 2013, metastases to the bones (pelvic and lumber vertebrae) and hilar lymph nodes were detected, and she underwent treatment with axitinib followed by sunitinib. In 2016, her mediastinal lymph nodes were treated with EBRT of 60 Gy in 30 fractions at an external institution. In May 2018, liver metastasis was detected, and this lesion was treated with radiofrequency ablation. In Sep-
Address for correspondence: Gen Suzuki, MD, PhD, Department of Radiology, Kyoto Prefectural University of Medicine, 465 Kajiicho Kawaramachi Hirokoji, Kamigyo-ku, Kyoto 602-8566 Japan, phone: +81 752515618, fax: +81 752515840, $₫$ e-mail: gensuzu@koto.kpu-m.ac.jp
Received: 22.08 .2019

Accepted: 08.10 .2019

Published: 30.10 .2019 
tember 2018, metastasis in the right ovary led to its surgical excision, followed by nivolumab at the standard dosage (infusions of $240 \mathrm{mg}$ every alternate week) as third-line therapy. Four months after the initiation of nivolumab, computed tomography (CT) imaging revealed the progression of lung, liver and bone metastases and nivolumab monotherapy was further continued for two months. In January and February 2019, she underwent radiofrequency ablation for newly discovered liver metastases. In March 2019, CT imaging demonstrated gradual growth of the left iliac bone metastasis. The Tumor Board at our hospital decided that there was no surgical indication for this lesion.

The CT scan acquired prior to her visit to our hospital revealed an iliac crest tumor measuring $9.4 \times 8.8 \times 6.0 \mathrm{~cm}$ (Figure 1A) and tumor volume measuring $347.5 \mathrm{~cm}^{3}$. She was referred to our department for treatment and considering the large tumor burden other than metastases, we proposed HDR-ISBT for tumor control and volume reduction. Informed consent was obtained from the patient and her family. Nivolumab was continued until 10 days before the initiation of brachytherapy. After administration of spinal anesthesia, 13 applicator catheters were percutaneously inserted into the tumor under real-time CT guidance. These applicators were placed so that the whole tumor area was irradiated with simultaneous controlling the position of the needle against the adjacent structures.

After completion of this procedure, CT scan was taken for planning radiation therapy. In the treatment planning system (Oncentra ${ }^{\circledR}$ Brachy; Elekta AB, Stockholm, Sweden), clinical target volume (CTV) was set up based on the acquired CT images and 7 Gy per fractionated dose was prescribed in $100 \%$ of the CTV. The doses encompassing $90 \%, 95 \%$, and $98 \%$ of the CTV $\left(\mathrm{D}_{90}, \mathrm{D}_{95}, \mathrm{D}_{98}\right)$ were $9.2 \mathrm{~Gy}$, $8.5 \mathrm{~Gy}$, and 7.9 Gy per fraction, respectively. The minimum dose to the most exposed $0.1 \mathrm{cc}, 1.0 \mathrm{cc}$, and $2.0 \mathrm{cc}$ $\left(\mathrm{D}_{0.1 \mathrm{cc}}, \mathrm{D}_{1 \mathrm{cc}}, \mathrm{D}_{2 \mathrm{cc}}\right)$ of the small bowel were $7 \mathrm{~Gy}, 6.5 \mathrm{~Gy}$, and 6.3 Gy per fraction, respectively. The HDR-ISBT of 35 Gy in five fractions for three days (two times a day with six hours' interval) was administered (Figure 1B).
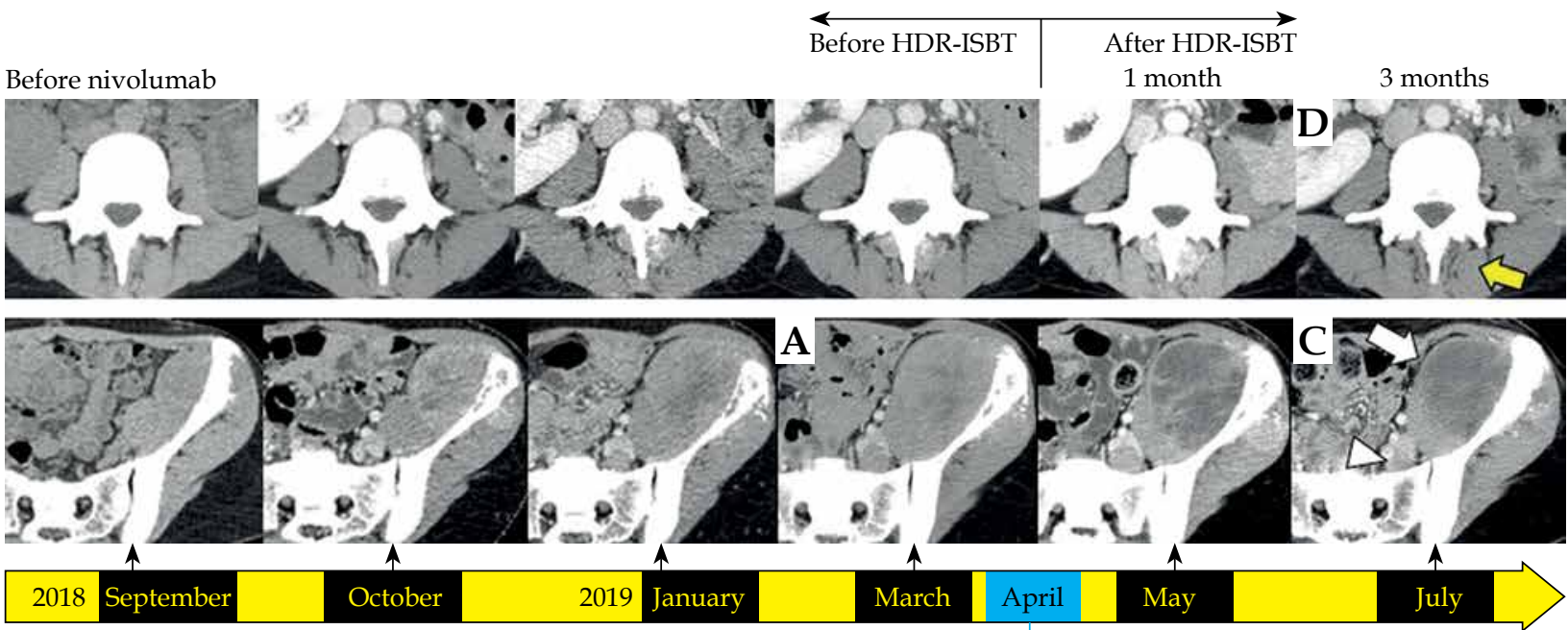

HDR-ISBT 35 Gy/5 fractions/3 days (1-3.04.2019)

B

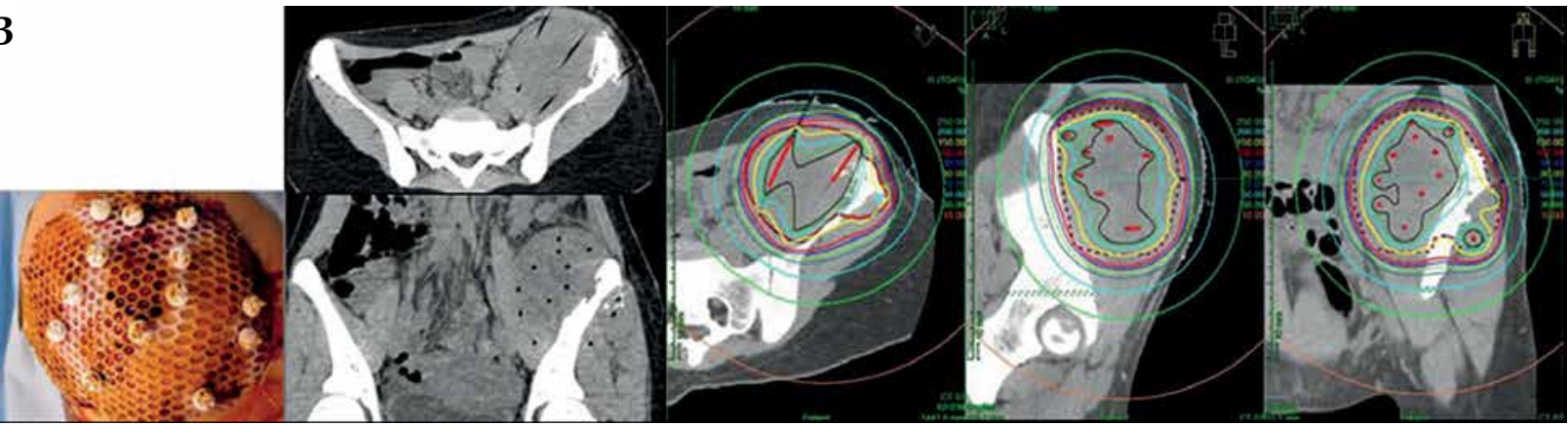

Fig. 1. Clinical course of treatment and computed tomography (CT) imaging: A) CT image taken one month before brachytherapy shows left iliac bone metastasis; B) Pelvis scan with high-dose-rate interstitial brachytherapy (HDR-ISRT) dosimetry and applicator reconstruction shows 13 applicator needles percutaneously inserted into the target, and 7 Gy per fraction dose was prescribed in $100 \%$ of clinical target volume; C) CT image taken three months after brachytherapy and resumption of nivolumab shows shrinkage of the left iliac lesion (white arrow) and the left internal iliac lymph node (white arrowhead); D) CT image taken three months after brachytherapy and resumption of nivolumab shows complete remission of the metastatic spine disease (yellow arrow) 
Brachytherapy was carried out using a ${ }^{192}$ Ir remote after-loading system (MicroSelectron v3 ${ }^{\circledR}$ HDR, Nucletron, ELEKTA AB, Stockholm, Sweden).

Nivolumab was restarted nine days after HDR-ISBT. Three months after completion of HDR-ISBT, shrinkage of the irradiated left iliac lesion (Figure 1C) and left internal iliac lymph node (Figure 1C) was evident by CT. Simultaneously, the metastatic lesion in the lumbar vertebrae $\left(\mathrm{L}_{4}\right)$, which was the non-irradiated site, showed evidence of disappearance on the same CT (Figure 1D). There was no apparent increase in size of the remaining non-irradiated metastatic lesions. Figure 1 presents the summarized imaging studies and clinical course after the initiation of nivolumab.

During the HDR-ISBT period, pain associated with applicator placement was addressed using continuous venous analgesia. The day after the applicator was placed, a significant elevated serum lactate dehydrogenase (LDH) level was observed in the blood test (Figure 2) that lasted for seven months. As we suspected tumor lysis syndrome, we performed intravenous rehydration, which led to a decrease in serum LDH levels within a few days, and the treatment was completed without any problems, and reached the normal range three months after brachytherapy (Figure 2, reference range 124-222 $\mathrm{U} / \mathrm{l})$. After the treatment, there were no complications except for mild dermatitis.

\section{Discussion}

Herein, we describe a 30-year-old Japanese female with intractable metastases of RCC who exhibited the abscopal effect after a combination treatment with nivolum$\mathrm{ab}$ and HDR-ISBT. To the best of our knowledge, this is the first report demonstrating an abscopal effect following brachytherapy for human malignancy.

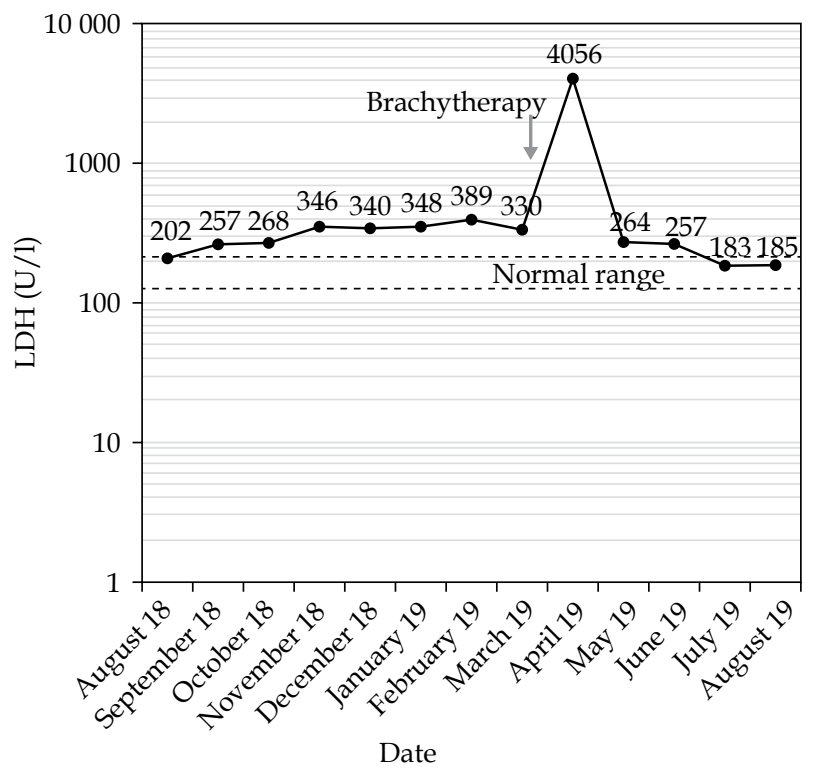

Fig. 2. High levels of serum lactate dehydrogenase (LDH) that persisted for seven months improved to normal levels, three months after brachytherapy
At a certain radiation dose, radiation-induced tumor cell damage is known to activate antitumor immune response through the release of tumor antigens and damage-associated molecular pattern, which, in turn, results in increased activation of antigen-presenting cells and T-lymphocytes. This activation of the immune system triggers antigen-specific, adaptive immunity, a phenomenon referred to as in situ radio-vaccination [6]. Several pre-clinical studies have demonstrated that regression of distant metastases, at remote non-irradiated sites of the body, termed the "abscopal effect", can be achieved by an appropriate timing and combination of radiation with immunotherapy $[7,8,9]$. This effect has been vetted in several clinical studies showing improved responses by combining radiation and immunotherapy in several types of malignant tumors $[1,2,3,4]$. However, nearly all preclinical and clinical studies evaluating radiation therapy in combination with immunotherapies have used EBRT, which typically, must pass through considerable volumes of normal tissue to reach the targeted tumor volumes. Circulating lymphocytes are highly sensitive to radiation therapy, with a $D_{90}$ of $0.5 \mathrm{~Gy}$ [10], and large treatment fields are viewed as a shortcoming in obtaining a clinically compelling response, leading to long-term lymphopenia in some cases [11]. Such effects may limit the efficacy of immune stimulatory agents in conjunction with EBRT. Pike et al. showed that extracranial or prolonged courses of radiation increase the risk of severe lymphopenia, which is associated with lesser survival than that of patients treated with ICI [12].

Various EBRT regimens and techniques, particularly conformal techniques such as intensity modulated radiation therapy (IMRT) or stereotactic body radiotherapy (SBRT), could play an important role. Contrary to SBRT/IMRT, radiation delivered using image-guided brachytherapy achieves unmatched dose conformality. This can be considered an advantage of brachytherapy compared with EBRT, because of the sparing of normal tissue and pertinent lymphoid organs from the low doses of radiation that may eradicate sensitive immune cell lineages. Moreover, heterogeneity of the radiation dose delivered to the target lesions may facilitate the ideal involvement of multiple immunogenic mechanisms, each with different dose response profiles [5]. In effect, regions closest to the source of a high dose of radiation exhibits greater tumor cell death than other regions, as observed in models of tumor response to radiation therapy [13]. Further, this region exhibits maximal immunogenic tumor cell death and release of tumor-specific antigens [14]. However, Vanpouille-Box et al. proposed not to deliver more than 12-14 Gy per fraction because of the potential activation of the $3^{\prime}$ repair exonuclease 1 (TREX1) [15]. TREX1 attenuates their immunogenicity by degrading DNA that accumulates in the cytosol upon radiation.

Outside the highest dose regions, high-intermediate dose per fraction (8-12 Gy) may optimally induce cytoplasmic release of double-stranded DNA and phenotypic changes in the expression of immune susceptibility markers on tumor cells that survived radiation $[15,16,17]$. Moderate dose per fraction (2-5 Gy) may potentiate the release of immune stimulatory cytokines, leading to enhanced tumor infiltration by immune cells [18]. Some 
studies have shown that immunological response can also be initiated by trauma to the site $[19,20]$. Butterfield's review on cancer vaccinations dwells upon how surgery and tumor-ablative procedures of various magnitudes can be considered as a sort of "cancer vaccine" [21]. In this respect, ISBT may offer significant advantages over EBRT in initiating the in situ vaccine effect.

Our case had bulky iliac bone metastasis from RCC with a known radio-resistance phenotype. In general, bulky disease represents an important challenging obstacle for all currently available treatment options: in most cases surgery and EBRT cannot achieve more than a palliative effect; SBRT is not indicated for such large tumors; whereas chemoradiotherapy would induce intolerable levels of toxicity. The advantage of brachytherapy compared with EBRT, regardless of the histological type, is that the maximum radiation dose can be delivered directly to the tumor without damaging healthy surrounding tissues, leading to better local control. In our case presented in this report, nivolumab combined with HDR-ISBT resulted in a marked reduction in irradiated as well as non-irradiated lesions, despite being refractory to prior treatments with nivolumab. In order to trigger the abscopal effect, it may be essential for the maximum size of the tumor lesion to be irradiated. Brachytherapy could favor the targeting of the maximal tumor size and/or repeat and locally treat several tumor sites. Multi-site irradiation increases the chances of successfully priming an antitumor immune response and might also destroy, or potentiate the destruction of resistant subclonal populations that could impair a complete response to ICIs $[22,23]$. Therefore, multi-site brachytherapy may be an emerging paradigm for treating metastatic disease in the future.

In our patient, the high level of serum LDH that lasted for 7 months improved to normal levels 3 months after brachytherapy (Figure 2). Recently, it has been shown that serum LDH may be correlated to the prognosis of RCC, suggesting that the serum LDH level can be used as a valuable biomarker for monitoring prognoses [24]. Although the mechanisms involved in radiation response need to be elucidated to better understand the true effect of brachytherapy, we believe that HDR-ISBT may further play an important role in enhancing the activity of ICIs across a broad range of malignancies.

\section{Conclusions}

This is the first case report demonstrating the abscopal effect following brachytherapy and immunotherapy for human malignancy and the evidence indicates that immuno-oncology effects are not limited to EBRT regimens as they can also be attained by brachytherapy.

\section{Disclosure}

The authors report no conflict of interest.

\section{References}

1. Leary R, Gardner RB, Mockbee C et al. Boosting abscopal response to radiotherapy with sargramostim: A review of data and ongoing studies. Cureus 2019; 11: e4276.
2. De Ruysscher D. Combination of radiotherapy and immune treatment: First clinical data. Cancer Radiother 2018; 22: 564-566.

3. Siva S, MacManus MP, Martin RF et al. Abscopal effects of radiation therapy: A clinical review for the radiobiologist. Cancer Lett 2015; 356: 82-90.

4. Dagoglu N, Karaman S, Caglar HB et al. Abscopal efect of radiotherapy in the immunotherapy era: Systematic review of reported cases. Cureus 2019; 11: e4103.

5. Patel RB, Baniel CC, Sriramaneni RN et al. Combining brachytherapy and immunotherapy to achieve in situ tumor vaccination: A review of cooperative mechanisms and clinical opportunities. Brachytherapy 2018; 17: 995-1003.

6. Herrera FG, Bourhis J, Coukos G. Radiotherapy combination opportunities leveraging immunity for the next oncology practice. CA Cancer J Clin 2017; 67: 65-85.

7. Meng X, Feng R, Yang L et al. The role of radiation oncology in immuno-oncology. Oncologist 2019; 24 (Suppl 1): S42-S52.

8. Ozpiskin OM, Zhang L, Li JJ. Immune targets in the tumor microenvironment treated by radiotherapy. Theranostics 2019; 9: 1215-1231.

9. Weichselbaum RR, Liang H, Deng L et al. Radiotherapy and immunotherapy: A beneficial liaison? Nat Rev Clin Oncol 2017; 14: 365-379.

10. Nakamura N, Kusunoki Y, Akiyama M. Radiosensitivity of CD4 or CD8 positive human T-lymphocytes by an in vitro colony formation assay. Radiat Res 1990; 123: 224-227.

11. Order SE. The effects of therapeutic irradiation on lymphocytes and immunity. Cancer 1977; 39: 737-743.

12. Pike LRG, Bang A, Mahal BA et al. The impact of radiation therapy on lymphocyte count and survival in metastatic cancer patients receiving PD-1 immune checkpoint inhibitors. Int J Radiat Oncol Biol Phys 2019; 103: 142-151.

13. Fowler JF. 21 years of biologically effective dose. Br J Radiol 2010; 83: 554-568.

14. Demaria S, Kawashima N, Yang AM et al. Immune-mediated inhibition of metastases after treatment with local radiation and CTLA-4 blockade in a mouse model of breast cancer. Clin Cancer Res 2005; 11: 728-734.

15. Vanpouille-Box C, Alard A, Aryankalayil MJ et al. DNA exonuclease Trex1 regulates radiotherapy-induced tumour immunogenicity. Nat Commun 2017; 8: 15618.

16. Yoshimoto Y, Suzuki Y, Mimura K et al. Radiotherapy-induced anti-tumor immunity contributes to the therapeutic efficacy of irradiation and can be augmented by CTLA-4 blockade in a mouse model. PLoS One 2014; 9: 1-8.

17. Cole WH. Efforts to explain spontaneous regression of cancer. J Surg Oncol 1981; 17: 201-209.

18. Liu SZ. Nonlinear dose-response relationship in the immune system following exposure to ionizing radiation: Mechanisms and implications. Nonlinearity Biol Toxicol Med 2003; 1: 71-92.

19. Lopez-Pastorini A, Plönes T, Brockmann M et al. Spontaneous regression of non-small cell lung cancer after biopsy of a mediastinal lymph node metastasis: A case report. J Med Case Rep 2015; 9: 2-5.

20. Esplin N, Fergiani K, Legare TB et al. Spontaneous regression of a primary squamous cell lung cancer following biopsy: A case report. J Med Case Rep 2018; 12: 65.

21. Butterfield LH. Cancer Vaccines. BMJ 2015; 3: h988.

22. Luke JJ, Lemons JM, Karrison TG et al. Safety and clinical activity of pembrolizumab and multisite stereotactic body radiotherapy in patients with advanced solid tumors. J Clin Oncol 2018; 36: 1611-1618.

23. Brooks ED, Chang JY. Time to abandon single-site irradiation for inducing abscopal effects. Nat Rev Clin Oncol 2019; 16: 123-135.

24. Shen J, Chen Z, Zhuang Q et al. Prognostic value of serum lactate dehydrogenase in renal cell carcinoma: A systematic review and meta-analysis. PLoS One 2016; 11: e0166482. 